\title{
Adhesion Energy and Roughness Observation on Alkali Treated Natural Fiber as Composites Reinforcement
}

\author{
Dody Ariawan ${ }^{1 *}$, Sunardi $^{2}$, H I Akbar ${ }^{3}$ \\ ${ }^{1}$ Department of Mechanical Engineering Faculty of Engineering Universitas Sebelas Maret, Surakarta, Indonesia \\ ${ }^{2}$ Department of Mechanical Engineering Faculty of Engineering Universitas Sultan Ageng Tirtayasa \\ ${ }^{3}$ Department of Mechanical Engineering Vocational School Universitas Sebelas Maret, Surakarta, Indonesia \\ *Corresponding author. Email: dodyariawan@staff.uns.ac.id
}

\begin{abstract}
This paper reviews the influence of alkali treatment to adhesion energy and roughness change of natural fiber in correlation to interfacial interaction between fiber and matrix. The reviews of alkali treatment effect to natural fiber were focused on surface roughness, adhesion properties and interfacial strength between fiber and matrix. The surface contour were observed by atomic force microscope (AFM) while adhesion properties were explained by AFM and contact angle analysis. The interaction between fiber and matrix was explained by interfacial shear strength tests results to support the discussion. The transformation in chemical, crystalinity index $(\mathrm{CrI})$, thermal stability and micrograph of fiber are presently discussed to support previous data.
\end{abstract}

\section{Keywords: Reinforcement, Natural Fiber, Adhesion Energy, Roughness}

\section{INTRODUCTION}

The interaction between matrix and natural fiber is strongly influenced the properties of fiber, such as its chemical and physical characteristics [1][2][3]. Various approaches have been carried out to improve the fibermatrix adhesion properties of natural fiber composites. Including chemical treatment of the matrix, fiber, or both, such as surface treatment. The surface treatment directly increases the wettability of the fiber by the matrix which results in an increase in the interfacial bond between both, that resulting in a composite with better mechanical properties [4]. Generally, silane, alkali, peroxide, and permanganate are used for chemical treatments for fiber surface modification [5][6][7]. However, alkali treatment is the most effective step to improve interfacial bonding compared to other chemical treatments [7].

Alkali treatment or mercerization has been proven by previous studies to be effective in increas-ing the adhesion among the cellulose fiber and the polymer matrix, resulting in an enhancement in me-chanical properties of the composite [5][6][9]. This treatment is soak the natural fibers in alkali solution. The effectiveness of this treatment depends on the solution, as well as the holding time and temperature of soaking the fibers [8]. Natural fiber treatment by $\mathrm{NaOH}$ encourages the ionization of hydroxyl to alkoxide. This treatment also reported able to rear-range the crystalline structure of cellulose fiber by transformation of monoclinic crystalline lattice of cellulose-I into cellulose-II crystalline structure [6][9]. More than that, the alkali treatment improves the interfacial behavior of the fiber surface as effect dis-appearance the lignin, pectin, waxy substances and natural oil that covering the surface of the fiber cell wall [10]. This promoted the interfacial adhesion through mechanical interlocking among the fibers and the matrix, and promotes friction between them. It resulted the higher mechanical characteristics of the composite.

The changes of chemical composition, crystallinity index, and micrographs of natural fiber after alkali treatment have been explained in other studies. The change of crystallinity and the removal of hemicellulose and impurities have been intensively observed by Fouier-Transform Infrared Spectrometer (FTIR) tests, Scanning Electron Microscope (SEM) micrograph, crystallinity index 
by X-Ray Diffraction (XRD) and X-ray Photoelectros Spectrocopy XPS test in previous studies [11][12][13][14]. Furthermore these studies also explained the mechanical improvement after treatment that supported other studies.

Previous studies reported the effectiveness of alkali treatment on fibers to enhance the strength and mechanical behavior of the composites. However, studies that focused on the modification of interfacial properties of treated fibers and its effect on the fiber-matrix bonding are limited to found. The previous studies revealed that the interfacial adhesion could be analized by the surface energy and adhesion energy measurement by contact angle and atomic force microscope observation, respectively [2][14][15][16]. The roughness measurement were conducted to support the results of surface energy measurement. It is necessary to know the analysis of the fiber surface roughness, so that the correlation between fiber treatment and mechanical properties is obtained. The comprehensive methods to analyze the correlation between alkali treatments on natural fiber in composites are discussed in this paper.

\section{CONTACT AND MEASUREMENT}

The contact angle method can be used effectively to obtain the surface energy of composite. Generally, the contact angle measurement using Wilhemy technique and fluid geometry analysis. Nevertheless, both methods are rarely applied to natural fiber that has irregularity surfaces and $p$ variation of perimeter. The modified Washburn method or capillary rise method can be used to measure wettability by surface adhesion of natural fibers [16]. The contact angle is formulated by the Washburn as seen in Eq.1 and Eq.2 [16].

$$
\begin{aligned}
& \cos \theta=\frac{m^{2}}{t} \frac{\eta}{\rho^{2} \gamma_{L} C} \\
& C=\frac{1}{2} \pi^{2} r_{C}{ }^{5} n_{C}{ }^{2}
\end{aligned}
$$

Where $\theta$ the solid/liquid contact angle, $m$ simbolized the weight of the liquid that penetrates into the capillary, $\eta$ the liquid viscosity, $\gamma_{L}$ is the liquid surface tension, $\rho$ the liquid density, and $t$ the penetration time. The parameter $C$ can be determined by assuming a mean capillary radius $r_{c}$ and a corresponding number of capillaries $n_{c}$ of the tube used in the experiment. The constant $C$ was determined by using a very low surface tension liquid (i.e. hexane), which fully wets the sample $(\theta=0)$.

The polar and disperse components of fiber surface energy are formulated by using the Owens-WendtRabel-Kaelble (OWRK), which allows for a linearization of the contact angles in the various liquids using Eq. 3 and Eq. 4 (Pietak, Korte et al. 2007)

$$
\begin{gathered}
-\frac{(1+\cos (\theta)) \gamma_{L}}{2 \sqrt{\gamma_{L}^{d}}}=\sqrt{\gamma_{S}^{p}} \sqrt{\frac{\gamma_{L}^{p}}{\gamma_{L}^{d}}}+\sqrt{\gamma_{S}^{d}} \\
y=-\frac{(1+\cos (\theta)) \gamma_{L}}{2 \sqrt{\gamma_{L}^{d}}} \quad \text { and } \quad x=\sqrt{\frac{\gamma_{L}^{p}}{\gamma_{L}^{d}}}
\end{gathered}
$$

Where $\gamma_{i}^{d}$ and $\gamma_{i}^{p}$ are simbolize the surface energy of disperse and polar components, respectively, with $i$ representing the index for solid $(S)$ or liquid $(L)$.

The analysis of contact angle was conducted on both of untreated and alkali treated fiber to provide the data of the contact angle and surface energy on the fiber. The total surface energy $(\gamma)$ are calculated by the sum of the polar energy $\left(\gamma^{p}\right)$, the dispersion energy $\left(\gamma^{d}\right)$ to evaluate the wettability properties of fiber. Previous studies used at least two different polarity of liquids need to be ascertained as seen in Table 1 [12][13]. The untreated kenaf fiber has a bigger contact angle with lower total surface energy compared to alkali-treated fiber, both in water and $50 \%$ ethylene glycol as shown in Table 2 . The improvement in the total surface energy of treated kenaf fiber is reported as the soaking time increases. It indicates that alkali treatment plays a significant role in boost the wettability of natural fiber. The results that obtained is correlated with previous works [2][17] on the alkali treated natural fiber with similar ranges of surface energy values.

Table 1. Polar and disperse components of surface energy of water and $50 \%$ ethylene glycol [13]

\begin{tabular}{|l|c|c|c|}
\hline Liquid & $\begin{array}{c}\gamma_{L}^{P}(\mathrm{~m} \mathrm{Nm} \\
1\end{array}$ & $\begin{array}{c}\gamma_{L}^{d}(\mathrm{~m} \\
\left.\mathrm{Nm}^{-1}\right)\end{array}$ & $\gamma_{L}\left(\mathrm{~m} \mathrm{Nm}^{-1}\right)$ \\
\hline Water & 43.70 & 28.10 & 72.80 \\
\hline $\begin{array}{l}50 \% \text { Eth- } \\
\text { ylene glycol }\end{array}$ & 37.89 & 20.00 & 57.89 \\
\hline
\end{tabular}

Untreated natural fibers have lower wettability towards polymeric matrix, it is due to the existence of waxes and lignin that covered fiber surface whereby lignin and wax components are non-polar while the cellulose is polar [1]. The removing non-polar compound by alkali treatment induces the increased polarity of the natural fiber which subsequently enhances the surface energy.

Furthermore, the roughness affects on the wettability as it may determine the dispersion properties of fiber surface on any liquid [18][19]. The higher roughness impede the proper dispersion of liquid in contact with the surface consequently enhances the contact angle [20][18]. Therefore, the untreated natural fiber exhibit low dispersion energy value owing to the removal of im 


Table 2. Contact angle, dispersion energy, polarity energy and surface energy of kenaf fiber in different times of
soaking in 6\% NaOH [12]
\begin{tabular}{|c|c|c|c|c|c|}
\hline Time of soaking $(\mathrm{h})$ & Contact Anglea & Contact Angle & $\begin{array}{c}\text { Dipersion } \\
\left(\mathrm{mN} \mathrm{m}^{-1}\right)\end{array}$ & Polarity $\left(\mathrm{mN} \mathrm{m}^{-1}\right)$ & $\begin{array}{c}\text { Total Surface En- } \\
\text { ergy }\left(\mathrm{mN} \mathrm{m}^{-1}\right)\end{array}$ \\
\hline 0 & $87.24 \pm 0.05$ & $75.25 \pm 0.01$ & 3.01 & 51.3 & 54.31 \\
\hline 1 & $85.62 \pm 0.01$ & $73.6 \pm 0.01$ & 3.07 & 53.32 & 56.39 \\
\hline 2 & $83.74 \pm 0.01$ & $70.54 \pm 0.05$ & 4.60 & 61.26 & 65.86 \\
\hline 3 & $82.62 \pm 0.03$ & $68.99 \pm 0.13$ & 4.81 & 63.51 & 68.31 \\
\hline 4 & $82.11 \pm 0.01$ & $68.44 \pm 0.05$ & 4.93 & 64.67 & 69.6 \\
\hline 5 & $81.72 \pm 0.02$ & $67.01 \pm 0.13$ & 9.89 & 77.81 & 87.7 \\
\hline
\end{tabular}

${ }^{a}$ In distilled water

${ }^{\mathrm{b}}$ In $50 \%$ ethylene glycol and 50\% distilled water

$\mathrm{a}, \mathrm{b}$ display the mean \pm standard deviation

purities on fiber surface after alkali treatment. The removal impurities decrease the surface roughness which will be discussed in the micrograph observation section. As a consequence, the decrease of roughness increasing the dispersion that raise the total surface energy as well as the wettability of alkali treated kenaf fiber [12].

To ensure the interaction among alkali-treated fiber with resin, the surface energy of cured resin could be determined using a similar method to calculate the surface energy of fiber. Minimal changes in surface energy of cured resin, when compared with the uncured resin, the studies reported that surface properties did not significantly change after the curing process. (Abbot et. al. 1988) reported that there are no significant changes for epoxy resin system on surface energy even with $50 \%$ curing conversion rate [21]. Based on the surface energy, the work of adhesion among resin and fiber is calculated with Owens-Wendt formula following Eq. 5 [22]

$$
W_{a}=2\left(\sqrt{\gamma_{S}^{d}} \gamma_{L}^{d}+\sqrt{\gamma_{S}^{p} \gamma_{L}^{p}}\right)
$$

Table 3. Work of adhesion of Kenaf Fiber/Acrodur composites.

\begin{tabular}{|l|c|}
\hline \multicolumn{1}{|c|}{ Composites } & $\begin{array}{c}\text { Work of Adhesion } \\
(\mathrm{mJ} / \mathrm{m})\end{array}$ \\
\hline Untreated Kenaf Fiber & 119.70 \\
\hline $\begin{array}{l}\text { Alkali Treated Kenaf Fiber in } 6 \% \\
\text { NaOH for } 3 \mathrm{~h}\end{array}$ & 153.21 \\
\hline $\begin{array}{l}\text { Alkali Treated Kenaf Fiber in } 6 \% \\
\mathrm{NaOH} \text { for } 3 \mathrm{~h} \text { at } 60^{\circ} \mathrm{C}\end{array}$ & 158.45 \\
\hline $\begin{array}{l}\text { Heat Treated Kenaf Fiber at } \\
140^{\circ} \mathrm{C} \text { for } 10 \mathrm{~h}\end{array}$ & 112.40 \\
\hline
\end{tabular}

Based on previous study [13], the alkali treatments enhanced the adhesion of fiber toward acrodur resin as shown in Table 3 that could give a guide to a better fiber

to matrix adhesion. It can be concluded that the alkali treatment might improve the wettability of the fiber during processing (uncured state) and improve fiber to matrix adhesion in cured composites.

\section{MICROGRAPH OBSERVATION}

The changes and topography of fiber surface after alkali treatment were observed with an atomic force microscope (AFM). AFM can generate three-dimensional topography of samples and can be used to explore the surface roughness of fiber. By using AFM, the surface roughness could be calculated to support dispersion data as be resulted by contact angle measurement. Futhermore, AFM could detect the adhesion energy that could be supported the OWRK calculation in contact angle measurement.

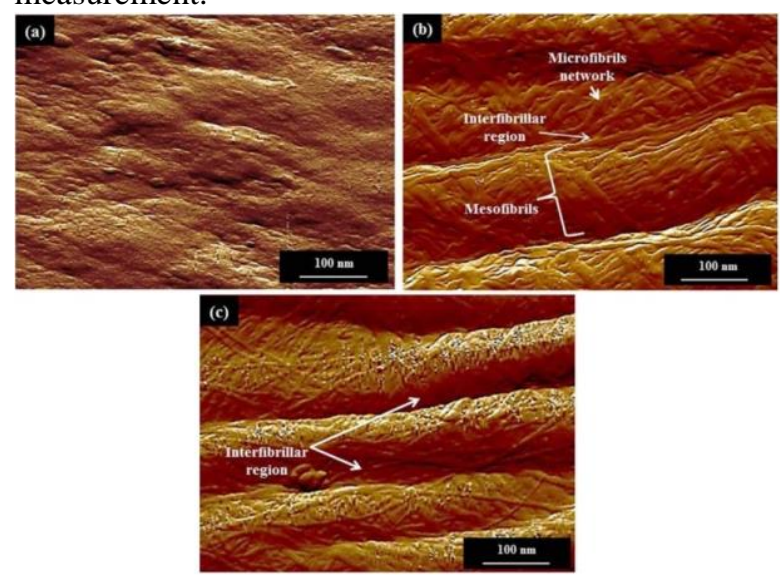

Figure 1 AFM images of untreated and treated kenaf fiber (a) Untreated kenaf fiber, (b) Kenaf fiber after treatment in $6 \% \mathrm{NaOH}$ for $3 \mathrm{H}$, (c) Kenaf Fiber after alkali treatment in $6 \% \mathrm{NaOH}$ at $60^{\circ} \mathrm{C}$ for 3 hours [13]. 
Figure 1 shows AFM images of untreated and treated kenaf fiber that showing the morphological changes on the kenaf fiber [13], while Fig. 2 displays the surface topography alterations post treatments [12]. From Fig. 1 (a), the untreated kenaf fiber result-ed nonuniform surface with globular topography, it due to the non-cellulosic cement and lignin [23]. The removal of the protective layer (on the primary cell wall) and the binding materials using alkali treatment has exposed the microfibril network on the fiber sur-face of kenaf fiber after treatment in $6 \% \mathrm{NaOH}$ for 3 hours and Kenaf Fiber after alkali treatment in $6 \% \mathrm{NaOH}$ at $60^{\circ} \mathrm{C}$ for 3 hours (Fig. $1 \mathrm{~b}$ and c). The diameter of the microfibril in the range of 1-20 nm was also found in the previous study [24]. Additional-ly, it is interesting to analyze that the microfibril net-work and interfibrillar region between the mesofibrils are more notable in the kenaf fiber after alkali treat-ment in $6 \% \mathrm{NaOH}$ at $60 \mathrm{oC}$ for 3 hours. As reported [5], the existing hemicellulose and lignin on the fiber surface could be removed in a more effective route under alkalization treatment at a higher temperature.

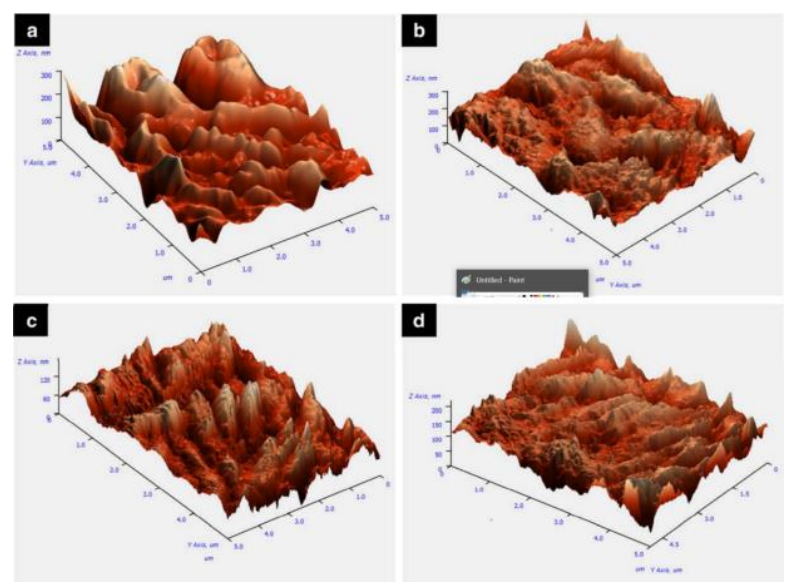

Figure 2 AFM results of kenaf fiber after alkali treatment in $6 \% \mathrm{NaOH}$ for a) $0 \mathrm{~h}, \mathrm{~b}) 1 \mathrm{~h}, \mathrm{c}) 3 \mathrm{~h}$, and d) $5 \mathrm{~h}$ [12].
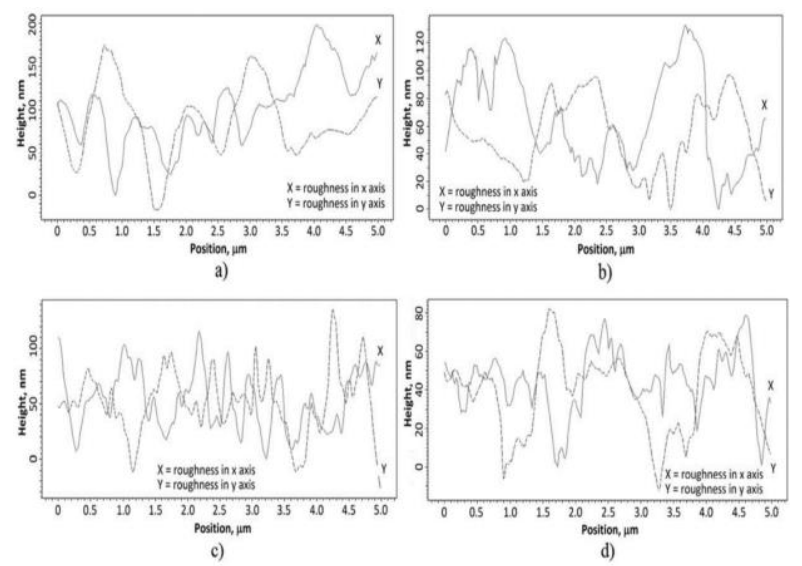

Figure 3 Roughness contour height of kenaf fiber after alkali treatment in $6 \% \mathrm{NaOH}$ for a) $0 \mathrm{~h}$, b) $1 \mathrm{~h}$, c) $3 \mathrm{~h}$, and d) $5 \mathrm{~h} \mathrm{[12].}$

Figure 2 shows the AFM topography of untreated and alkali- treated kenaf fiber in isometric view and Fig. 3 displays the roughness as reported [12]. The untreated kenaf fiber (Fig. 2a) has fine surfaces with globular substructures and high peak contour (Fig 3a). That structures are due to the existence of waxes, fats, lignin, pectin and hemicellulose on the outer surface of the fiber [17]. In contrast, the alkali-treated kenaf fiber (Fig. 2 (b-d)) shows flaky and grooved-like surfaces with low peak contour (see Fig 3 (b-d)) as a result of removal of cementing composund. Surface analysis of the kenaf fiber can be represented with the roughness of root mean square (RMS) value and area peak density as shown in Table 4.

Table 4. Roughness properties of kenaf fiber surface after alkali treatment in different temperature [13]

\begin{tabular}{|l|c|c|}
\hline Treatment & $\begin{array}{c}\text { Roughness, } \\
\text { RMS }(\mathrm{nm})\end{array}$ & $\begin{array}{c}\text { Area Peak } \\
\text { Density }\left(\mu \mathrm{m}^{-1}\right)\end{array}$ \\
\hline Untreated & 94.15 & 10.600 \\
\hline $6 \% \mathrm{NaOH}$ for $3 \mathrm{~h}$ & 25.46 & 43.056 \\
\hline $\begin{array}{l}6 \% \mathrm{NaOH} \text { for } 3 \mathrm{~h} \\
\text { in } 60^{\circ} \mathrm{C}\end{array}$ & 23.58 & 49.197 \\
\hline
\end{tabular}

The roughness root means square (RMS) and area peak density of the kenaf fiber are shown in Table 4. Through AFM, the surface roughness of kenaf fiber is calculated by means of the RMS (an average value of profile height from the mean line recorded within the evaluation of length) using Eq. 6, where $\mathrm{z}$ symbolize the vertical height of the surface, while $z_{n}$ symbolize the mean value of the surface height relative to the plane.

$$
R_{r m s}=\sqrt{\sum_{n=1}^{N} \frac{(Z n-Z)^{-2}}{N}}
$$


Untreated kenaf fiber surfaces shown higher RMS compared to alkali-treated kenaf fiber. The results indicate that the surface roughness of kenaf fiber decreases, due to the removal of low molecular densi-ty material with alkali treatment. On the other hand, the area peak density shows an improvement as al-kali treatment to the kenaf fiber. Area peak density refers to the number of peaks per unit area. Longer soaking time resulted in increasing the number of peaks on the kenaf fiber surface which provides higher fiber-matrix specific contact area and leads to enhancement of fiber wettability.

Figure 4 show the adhesion energy values were taken by a pixel analysis the noncontact mode AFM using the NX10 Park Systems. Alkali treatment of the fiber significantly improved the adhesion energy of kenaf fiber from $70.98 \mathrm{fJ}$ to $115.52 \mathrm{fJ}$ [11].
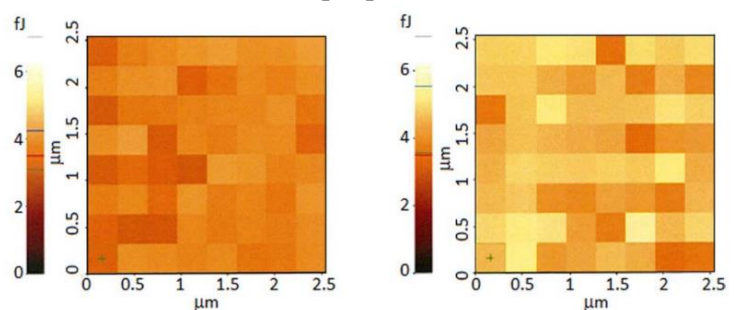

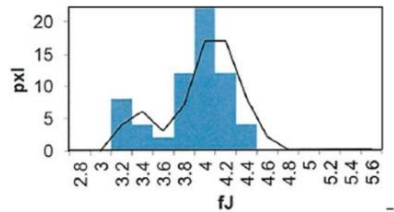

a)

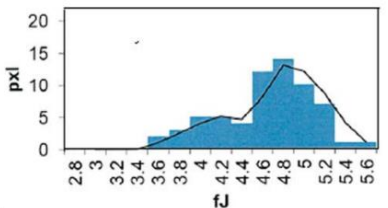

b)
Figure 4 Adhesion energy distribution and histogram of AFM results of (a) untreated kenaf fiber and (b) alkali treated kenaf fiber in $6 \% \mathrm{NaOH}$ for $3 \mathrm{~h}$ ([11].

The increase of adhesion energy is related to the removal of impurities, hemicellulose and lignin, that expose the polar cellulose on the kenaf fiber under alkalization treatment. Even though the RMS values increased, the surface of the alkali-treated fiber was more flat, that it had lower peak height values as seen in Fig. 2 and 3. The increasing adhesion energy of the alkali-treated kenaf fiber is due to the improving interaction among fiber and matrix, which also impacted the contact angle and surface energy. Contact angle calculating was used to evaluate the interfacial properties of the kenaf fiber towards the unsaturated polyester resins (UPR), in terms of surface energy characterization. In general, the contact angle is affected by the surface roughness, fibers hygroscopicity, and the chemical constituents in the fiber i.e. cellulose, lignin, hemicellulose, wax, pectin, etc [1]

\section{CONCLUSION}

Alkali treatment effectively removed the impurities and cementing material on natural fiber surface. The removal of non-polar substances of the natural fiber surface contributed to the increased of relatively high total surface energies of the alkali treated fiber. Total surface energy is merely the sum of the polar energy and the dispersion energy that could be calculated by using contact angle measurement technique. Higher surface energies of alkali treated fiber than the surface energy of untreated fiber promoted better wettability of the fibers in the matrix that has low surface energy. These improved interfacial bonding between matrix and natural fiber. Atomic Force Microscope (AFM) micrographs could provide the obvious evidence of these removal and showed the decrease of roughness. Furthermore, AFM results indicated the increase of area peak density that relate to the improvement of the dispersed bonding energy in alkali treated fiber along with the total surface energy of fiber.

\section{REFERENCES}

[1] Gassan, J., et al 2000 Macromol. Mater. Eng. 283(1) pp 132-139.

[2] Pietak, A., et al. 2007 Appl. Surf. Sci. 253(7) pp 3627-3635.

[3] Diharjo, K., et al. 2017 AIP Conf. Proc. 1788, 030050.

[4] Azwa, Z., et al. 2013 Mater. Des. 47 pp 424-442.

[5] Rong, M. Z., et al. 2001 Compos. Sci. Technol. 61(10) pp 1437-1447.

[6] John, M. J. and R. D. Anandjiwala 2008 Polym. Compos. 29(2) pp 187-207.

[7] Williams, T., et al. 2011 Inter. J. Polym. Sci. 2011.

[8] George, J., et al. 2004 Polym. Eng. \& Sci. 41(9) pp 1471-1485.

[9] Hashim, M. Y., et al. 2012 Proc. Wor. Acad. Sci. Eng. Tech.

[10] Mwaikambo, L. Y. and M. P. Ansell 2002 J. Appl. Polym. Sci. 84(12) pp 2222-2234.

[11] Ariawan, D., et al. 2016 Polym. Compos. 37(12) pp 3516-3526.

[12] Ariawan, D., et al. 2017 Polym. Compos. 38(3) pp 507-515.

[13] Salim, M. S., et al. 2019 Polym. Compos. 40(S1) pp E214-E226.

[14] Ariawan, D., et al. 2020 Alex. Eng. J. 59(5) pp 3981 3989.

[15] Rebouillat, S., et al. 1999 Inter. J. Adhes. Adhes. 19(4) pp 303-314.

[16] Aranberri-Askargorta, I., et al. 2003 J. Colloid Interfacial Sci. 263(2) pp 580-589.

[17] Doan, T.-T.-L., et al. 2012 Compos. Sci. Technol. 
72(10) pp 1160-1166.

[18] Kubiak, K., et al. 2011Wear 271(3) pp 523-528.

[19] Svetovoy, V. and G. Palasantzas 2014 Adv. Colloid Interface Sci. 216 pp 1 - 19.

[20] Meiron, T. S., et al. 2004 J. Colloid Interfacial Sci. 274(2) pp 637-644.

[21] Abbott, T. P., et al. 1988 J. Wood Chem. Technol. 8(1) pp 1-28.

[22] Tran, L. Q. N., et al. 2013 Compos. Sci. Technol. 80 pp 23-30

[23] Balnois, E., et al. 2007 Compos. Interfaces 14(7-9) pp 715-731.

[24] Dittenber, D. B. and H. V. GangaRao 2012 Compos. -A: Appl. Sci. Manuf. 43(8) pp 1419-1429. 